\title{
Analyzing the role of adjuvant or salvage radiotherapy for spinal myxopapillary ependymomas
}

\author{
Rupesh Kotecha, MD, ${ }^{1,2}$ Martin C. Tom, MD, ${ }^{3}$ Mihir Naik, DO, ${ }^{3}$ Lilyana Angelov, MD, ${ }^{4}$ \\ Edward C. Benzel, MD, ${ }^{4}$ Chandana A. Reddy, MS, ${ }^{3}$ Richard A. Prayson, MD, MEd, ${ }^{5}$ lain Kalfas, MD, ${ }^{4}$ \\ Richard Schlenk, MD, ${ }^{4}$ Ajit Krishnaney, MD, ${ }^{4}$ Michael P. Steinmetz, MD, ${ }^{4}$ William Bingaman, MD, ${ }^{4}$ \\ John H. Suh, MD, ${ }^{3}$ and Samuel T. Chao, MD ${ }^{3}$
}

\begin{abstract}
1Department of Radiation Oncology, Miami Cancer Institute, Baptist Health South Florida, Miami; ${ }^{2}$ Herbert Wertheim College of Medicine, Florida International University, Miami, Florida; ${ }^{3}$ Department of Radiation Oncology, Taussig Cancer Institute, Cleveland Clinic; and Departments of ${ }^{4}$ Neurological Surgery and ${ }^{5}$ Anatomic Pathology, Cleveland Clinic, Cleveland, Ohio
\end{abstract}

OBJECTIVE The authors sought to describe the long-term recurrence patterns, prognostic factors, and effect of adjuvant or salvage radiotherapy (RT) on treatment outcomes for patients with spinal myxopapillary ependymoma (MPE).

METHODS The authors reviewed a tertiary institution IRB-approved database and collected data regarding patient, tumor, and treatment characteristics for all patients treated consecutively from 1974 to 2015 for histologically confirmed spinal MPE. Key outcomes included relapse-free survival (RFS), postrecurrence RFS, failure patterns, and influence of timing of RT on recurrence patterns. Cox proportional hazards regression and Kaplan-Meier analyses were utilized.

RESULTS Of the 59 patients included in the study, the median age at initial surgery was 34 years (range 12-74 years), 30 patients $(51 \%)$ were female, and the most common presenting symptom was pain $(n=52,88 \%)$. Extent of resection at diagnosis was gross-total resection (GTR) in 39 patients (66\%), subtotal resection (STR) in 15 (25\%), and unknown in 5 patients $(9 \%)$. After surgery, 10 patients (17\%) underwent adjuvant RT (5/39 GTR [13\%] and 5/15 STR [33\%] patients). Median follow-up was 6.2 years (range $0.1-35.3$ years). Overall, 20 patients (34\%) experienced recurrence (local, $n=$ 15 ; distant, $\mathrm{n}=5$ ). The median RFS was 11.2 years ( $95 \% \mathrm{Cl} 77$ to not reached), and the 5 - and 10-year RFS rates were $72.3 \%(95 \% \mathrm{Cl} 59.4-86.3)$ and $54.0 \%(95 \% \mathrm{Cl}, 36.4-71.6)$, respectively.

STR was associated with a higher risk of recurrence $(\mathrm{HR} 6.45,95 \% \mathrm{Cl} 2.15-19.23, \mathrm{p}<0.001)$ than $\mathrm{GTR}$, and the median RFS after GTR was 17.2 years versus 5.5 years after STR. Adjuvant RT was not associated with improved RFS, regardless of whether it was delivered after GTR or STR. Of the 20 patients with recurrence, $12(60 \%)$ underwent salvage treatment with surgery alone (GTR, $n=6), 4(20 \%)$ with RT alone, and $4(20 \%)$ with surgery and RT. Compared to salvage surgery alone, salvage RT, with or without surgery, was associated with a significantly longer postrecurrence RFS (median 9.5 years vs 1.6 years; log-rank, $p=0.006$ ).

CONCLUSIONS At initial diagnosis of spinal MPE, GTR is key to long-term RFS, with no benefit to immediate adjuvant RT observed in this series. RT at the time of recurrence, however, is associated with a significantly longer time to second disease recurrence. Surveillance imaging of the entire neuraxis remains crucial, as distant failure is not uncommon in this patient population.

https://thejns.org/doi/abs/10.3171/2020.2.SPINE191534

KEYWORDS radiotherapy; spinal ependymoma; myxopapillary ependymoma; oncology

$\mathrm{S}$ PINAL myxopapillary ependymomas (MPEs) represent a clinically and biologically distinct subset of ependymomas, which are WHO grade I and tend to occur in the conus medullaris, cauda equina, and/or filum terminale. These tumors are rare, with a reported incidence of 1 per million person-years, and have a slight predominance in males and Caucasians. ${ }^{1}$ The natural history of spinal MPE is long, with reported 10-year overall and progression-free survival rates of $92 \%$ and $61 \%$, respectively. ${ }^{2}$

ABBREVIATIONS GTR = gross-total resection; MDACC = MD Anderson Cancer Center; MPE = myxopapillary ependymoma; RFS = relapse-free survival; RT = radiotherapy; STR = subtotal resection.

SUBMITTED December 21, 2019. ACCEPTED February 20, 2020.

INCLUDE WHEN CITING Published online May 1, 2020; DOI: 10.3171/2020.2.SPINE191534. 
Owing to the rarity of spinal MPEs, the optimal management remains unclear. Retrospective studies suggest that gross-total resection (GTR) is the most important factor in achieving long-term disease control. ${ }^{2-4}$ The role of adjuvant radiotherapy (RT) is less clear, with some studies suggesting benefit to upfront combined modality treatment regardless of the extent of resection. ${ }^{2,3,5-7}$ The objective of the current study was to assess the long-term clinical outcomes of consecutive patients managed at our institution, as well as to analyze recurrence patterns, prognostic factors, and the effect of adjuvant or salvage RT.

\section{Methods}

We queried an IRB-approved database at our tertiary institution for all patients with histologically confirmed spinal MPE treated consecutively between 1974 and 2015. Patient characteristics, tumor location and details, and treatment course at initial diagnosis and relapse, as well as all disease outcomes, were abstracted from the shared medical record.

All resections were piecemeal, owing to the difficulty in removing MPE in an en bloc fashion and the institutional practice at the time. Extent of resection was defined as either GTR or subtotal resection (STR), based on surgeon assessment rather than postoperative imaging due to the difficulty in distinguishing residual disease from postoperative changes. RT was delivered following primary resection or at disease recurrence, at the discretion of the treating physicians. RT was delivered via an external beam approach, using various techniques, such as $2 \mathrm{D}$ anterior-posterior fields, 3D conformal RT, and intensitymodulated RT, based on era of treatment and physician discretion.

The primary endpoint was relapse-free survival (RFS), calculated from the date of initial surgery to either the date of imaging confirmation of recurrence or the date of last clinical follow-up for patients who did not experience recurrence of their disease. The secondary endpoint was postrecurrence RFS, which was calculated from the date of initial recurrence to the date of subsequent recurrence or the date of last clinical follow-up for patients who did not experience a second recurrence.

\section{Statistical Analysis}

Cox proportional hazards regression was used to identify clinical factors associated with RFS on univariate analysis, as well as multivariable analysis when appropriate. The Kaplan-Meier method was used to estimate survival, and the log-rank test was used to compare survival curves. Statistical analysis was performed using SAS v9.4 (SAS Institute), and $p$ values $<0.05$ were considered statistically significant.

\section{Results}

The patient characteristics of the 59 patients who met inclusion criteria for this study are summarized in Table 1 . The median age at initial surgery was 34 years (range 12-74 years), and 30 patients (51\%) were female. The most common symptoms at initial presentation were pain in
TABLE 1. Patient and treatment characteristics

\begin{tabular}{|c|c|}
\hline Characteristic & No. $(\%)$ \\
\hline \multicolumn{2}{|l|}{ Age, yrs } \\
\hline$\leq 35$ & $33(56)$ \\
\hline$>35$ & $26(44)$ \\
\hline \multicolumn{2}{|c|}{ Karnofsky Performance Scale score } \\
\hline$\geq 70$ & $53(90)$ \\
\hline$<70$ & $6(10)$ \\
\hline \multicolumn{2}{|l|}{ Sex } \\
\hline Female & $30(51)$ \\
\hline Male & $29(49)$ \\
\hline \multicolumn{2}{|l|}{ Symptoms at presentation } \\
\hline Pain & $52(88)$ \\
\hline Numbness & $15(25)$ \\
\hline Weakness & $10(17)$ \\
\hline Abnormal gait & $9(15)$ \\
\hline Urinary incontinence & $6(10)$ \\
\hline Bowel incontinence & $5(8)$ \\
\hline Tingling & $2(3)$ \\
\hline \multicolumn{2}{|c|}{ Extent of resection at initial surgery } \\
\hline GTR & $39(66)$ \\
\hline STR or biopsy & $15(25)$ \\
\hline Not documented & $5(9)$ \\
\hline \multicolumn{2}{|c|}{ Adjuvant RT after initial surgery } \\
\hline No & $49(83)$ \\
\hline Yes & $10(17)$ \\
\hline \multicolumn{2}{|c|}{ Any recurrence after initial surgery } \\
\hline No & $39(66)$ \\
\hline Yes & $20(33)$ \\
\hline \multicolumn{2}{|c|}{ Salvage treatment for initial recurrence } \\
\hline RT alone & $4(20)$ \\
\hline RT and surgery & $4(20)$ \\
\hline Surgery alone & $12(60)$ \\
\hline
\end{tabular}

52 patients (88\%), numbness in $15(25 \%)$, weakness in 10 $(17 \%)$, and abnormal gait in 9 patients (15\%). A majority of patients underwent MRI for diagnosis (43 patients, 85\%) and the remainder had a combination of myelograms along with other diagnostic imaging, with 2 patients $(3.4 \%)$ assessed with CT only. Two-thirds of patients $(n=39,66 \%)$ in this series were diagnosed with disease in the lumbosacral region, and the remainder of patients $(n=20,34 \%)$ had disease in the thoracic spine.

Extent of resection at diagnosis was GTR in $39(66 \%)$, STR in $15(25 \%)$, and unknown in $5(9 \%)$ patients. After the initial surgery, 10 patients (17\%) underwent adjuvant RT (5 patients after GTR and 5 patients after STR) to a median dose of $49 \mathrm{~Gy}$ (range 45-58 Gy) delivered via conventional fractionation. Of these 10 patients, 7 had disease in the lumbosacral region and 3 had disease in the thoracic region. At last follow-up, 9 patients $(15 \%)$ had experienced a single recurrence, $7(12 \%)$ had 2 recurrences, 2 patients $(34 \%)$ had 3 recurrences, and 2 patients (34\%) had 4 recurrences. 


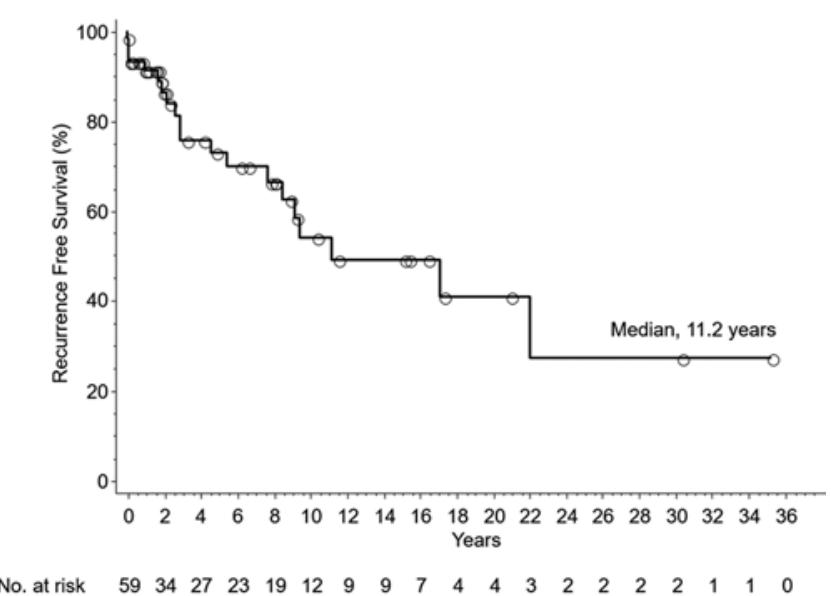

FIG. 1. RFS of all patients.

Median follow-up from time of initial surgery was 6.2 years (range $0.1-35.3$ years). Overall, 20 patients (34\%) experienced disease recurrence (local, $\mathrm{n}=15$; distant, $\mathrm{n}=5$ ). Among all patients, the median RFS was 11.2 years $(95 \%$ CI 7.7 to not reached), and the 5- and 10-year RFS rates were $72.8 \%$ (95\% CI 59.4-86.3) and 54.0\% (95\% CI 36.471.6), respectively (Fig. 1). The median survival time from initial surgery was 6.6 years (range $0.1-35.3$ years), and at the time of this analysis 54 patients $(92 \%)$ were alive and continued to be followed.

On univariate analysis (Table 2), STR was associated with a higher risk of disease recurrence (HR 6.45, 95\% CI 2.15-19.23, $\mathrm{p}<0.001$ ) compared to GTR. The 5-year RFS after GTR was $81.6 \%$ (95\% CI 66.6-96.7) versus 50.7\% (95\% CI 21.9-79.6) after STR (log-rank, p < 0.001; Fig. 2). Among all patients, adjuvant RT was not associated with improved RFS (log-rank, p = 0.97; Fig. 3). When analyzed separately by the type of resection, adjuvant RT was not associated with improved RFS after either GTR (log-rank, $\mathrm{p}=0.92)$ or STR (log-rank, $\mathrm{p}=0.27)$. Age $\leq 35$ years, disease location within the spine (i.e., thoracic vs lumbosacral), and adjuvant RT dose $\geq 50$ Gy were not associated with RFS ( $p>0.05$ for each).

Overall, 20 patients (34\%) had a recurrence: 15 patients (75\% of this subgroup) had a local recurrence and 5 patients (25\% of this subgroup) had a distant recurrence (recurrence at a different spinal segment). At the time of recurrence, 12 patients (60\%) subsequently underwent salvage treatment with surgery alone (GTR, $\mathrm{n}=6), 4(20 \%)$ with RT alone, and 4 patients (20\%) with both surgery and

TABLE 2. Univariate analysis for RFS

\begin{tabular}{lcc}
\hline \multicolumn{1}{c}{ Factor } & p Value & HR $(95 \% \mathrm{Cl})$ \\
\hline Age, yrs $(>35$ vs $\leq 35)$ & 0.49 & $1.37(0.56-3.40)$ \\
\hline STR vs GTR $(\mathrm{n}=54)$ & $<0.001$ & $6.45(2.15-19.23)$ \\
\hline T spine vs LS spine & 0.66 & $1.24(0.48-3.21)$ \\
\hline Adjuvant RT (no vs yes) & 0.97 & $1.02(0.34-3.09)$ \\
\hline Dose $(<50$ vs $\geq 50$ Gy, $n=10)$ & 0.15 & $0.17(0.01-1.92)$ \\
\hline
\end{tabular}

LS = lumbosacral; $T=$ thoracic.

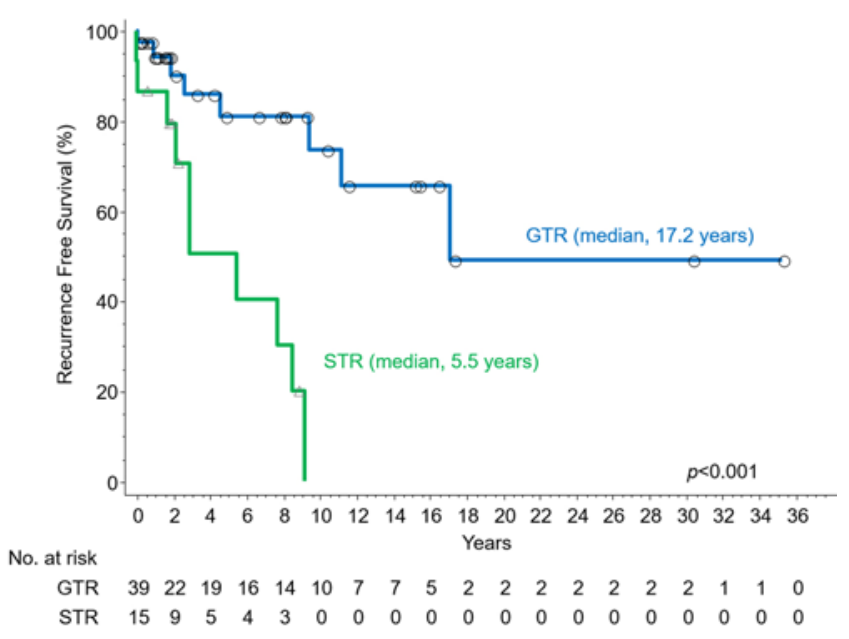

FIG. 2. RFS stratified by extent of resection. Figure is available in color online only.

RT. Compared to salvage surgery alone, salvage RT with or without surgery was associated with a significantly longer postrecurrence RFS (median 9.5 years vs 1.6 years; $\log$-rank, $\mathrm{p}=0.006$; Fig. 4 upper). The 5-year postrecurrence RFS was $27.5 \%$ (95\% CI 1.1-54.0) versus $84.6 \%$ (95\% CI 56.9-100) for patients treated with surgery alone versus RT, with or without surgery, respectively. We separately analyzed the subset of patients who were treated with surgery alone at first diagnosis but who received RT (with or without surgery) at the time of disease relapse (n $=8$ ). For these patients, there was a trend to improvement in time to next recurrence in the salvage setting exceeding the disease-free interval observed from initial diagnosis (median 9.5 vs 2.9 years; log-rank, p = 0.061; Fig. 4 lower). Of the patients who received salvage RT (with or without surgery), none had received prior RT. No other clear selection bias for the choice of salvage treatment was noted with regard to initial extent of resection, recurrence location (distant vs local), extent of resection at recurrence, RFS, or age at recurrence (Supplemental Tables 1 and 2).

Of the 5 patients with initial distal recurrence, 2 had re-

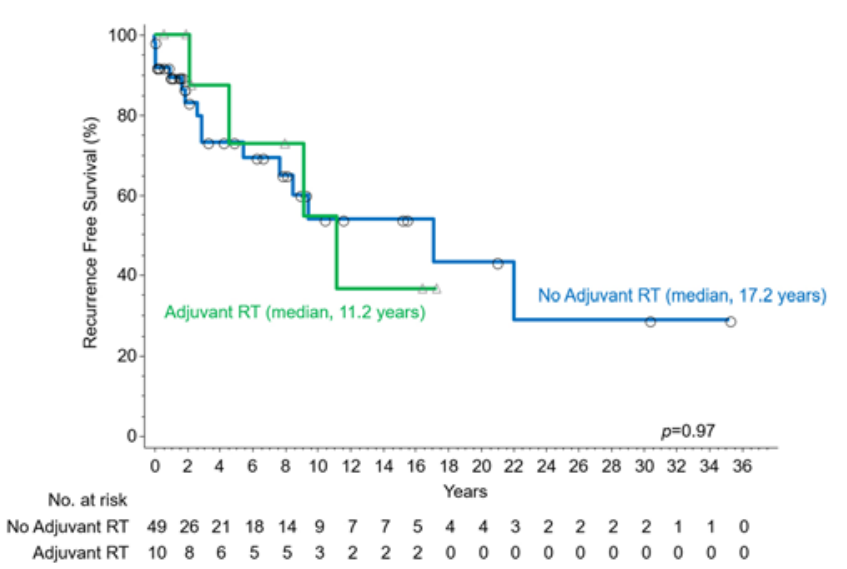

FIG. 3. RFS stratified by treatment with adjuvant RT. Figure is available in color online only. 


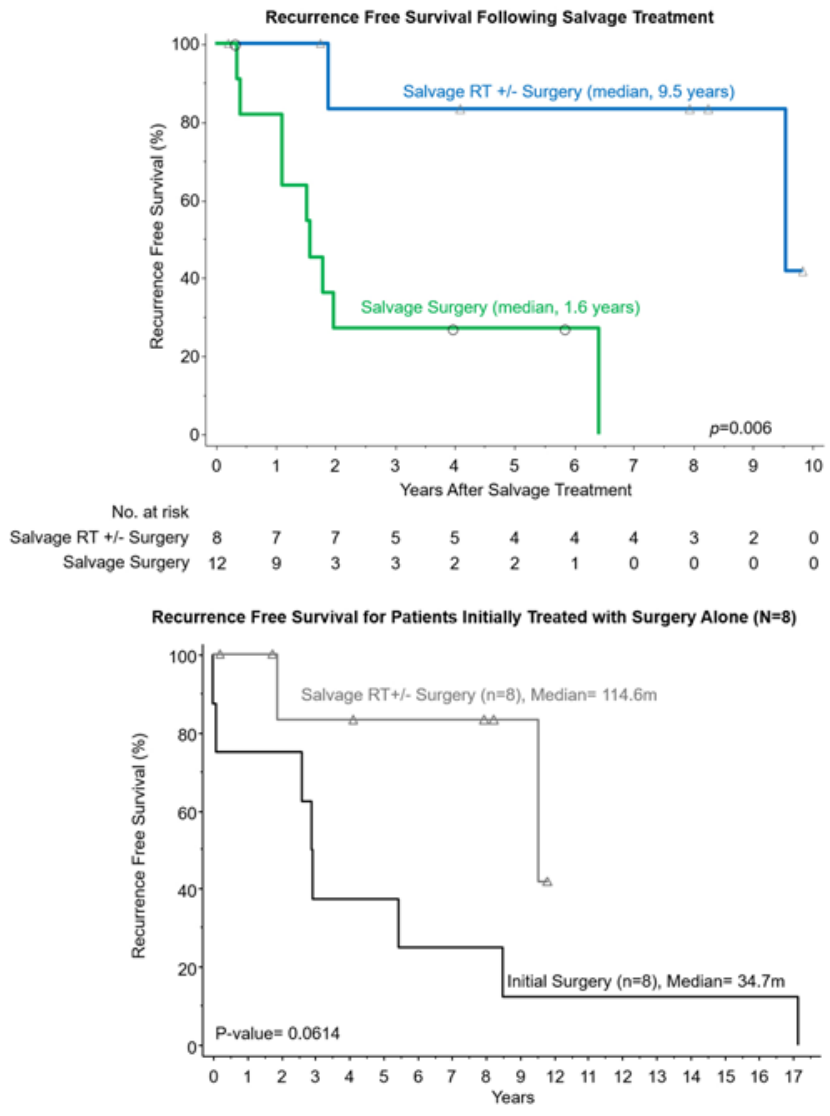

FIG. 4. Upper: RFS after salvage stratified by treatment with RT. Lower: RFS for patients treated with surgery alone and who received $\mathrm{RT}$ at the time of relapse; the same patients are plotted on both survival curves. Figure is available in color online only.

ceived initial adjuvant $\mathrm{RT}(\mathrm{GTR}, \mathrm{n}=1 ; \mathrm{STR}, \mathrm{n}=1)$, while the other 3 had received initial surgery alone (GTR, $n=$ 2 ; STR, $\mathrm{n}=1$ ). Distal failure was located in the inferioradjacent cord in 2 patients, inferior-nonadjacent cord in 1 , superior-adjacent cord in 0 , and superior-nonadjacent cord in 2 patients. Of the 15 patients with an initial local recurrence, 2 had received adjuvant RT while the remainder received initial surgery alone. Two of these patients went on to a subsequent distant recurrence: 1 patient in the superior-nonadjacent cord and 1 patient in the brain (nonadjacent).

\section{Discussion}

Management of spinal MPE represents a unique clinical challenge owing to the difficulty in achieving a complete en bloc resection, disease- and treatment-related morbidity associated with anatomical location, risk of locoregional and distant recurrence, rarity of this disease, and the relatively young age at disease presentation. Given the paucity of data in the literature with significant heterogeneity among analyses, additional data are sorely needed. Our present study of spinal MPE identified several key findings which supplement our understanding of this rare disease. First, initial GTR offers the best chance of cure, and has better outcomes than upfront combined modality therapy. Second, adjuvant RT after initial surgery is not associated with improved RFS. However, RT salvage therapy is associated with significant and dramatic improvements and prevention of a second disease relapse. Lastly, given the long-term follow-up in this series, we demonstrated that up to one-fourth of patients suffer treatment failure elsewhere in the spine, a finding that highlights the potential for late neuraxis failure in these patients.

Resection is the primary management strategy for spinal MPE, and GTR is associated with significantly improved disease outcomes. ${ }^{2,4,8}$ The frequency of GTR varies between $27 \%$ and $100 \%$ in the literature, and the data presented in our study demonstrate a clear benefit in terms of RFS with aggressive upfront GTR. ${ }^{8}$ Of course, in general, no surgeon undertakes a deliberate STR when a GTR is feasible, and therefore, even a definitive conclusion regarding GTR versus STR is difficult to promulgate in such a retrospective series. In our initial analysis of 37 patients with spinal MPE published in 2011, GTR demonstrated a numerically improved median RFS of 11.2 years compared to 5.5 years with STR, results which did not meet statistical significance (log-rank, $\mathrm{p}=0.075) .{ }^{9} \mathrm{How}-$ ever, in the present study with updated analysis, additional patients, and longer follow-up, GTR was associated with a statistically significant improvement in median RFS of 17.2 years compared to 5.5 years with STR. These data are consistent with the largest series of spinal MPE patients, performed using pooled data from MD Anderson Cancer Center (MDACC) and the Rare Cancer Network, which demonstrated in multivariable analysis that STR was associated with adverse local control (LC; HR 2.08, 95\% CI 1.13-3.82, $\mathrm{p}=0.01$ ) and progression-free survival (HR 2.14, 95\% CI 1.38-4.24, $\mathrm{p}=0.02){ }^{2}$ An older study similarly demonstrated that GTR was associated with improved overall survival (OS; median 19 years with GTR vs 14 years with STR). ${ }^{4}$ Furthermore, a large review of the literature with aggregated data from 1602 patients demonstrated a strong association with GTR and improved OS at 5 and 10 years. ${ }^{8}$ Together, these data collectively highlight the importance of obtaining a radical complete resection of spinal MPE and support the role of multidisciplinary management and specialized surgical expertise among newly diagnosed patients.

The role of adjuvant RT following initial resection is controversial, as this has never been prospectively evaluated in a randomized controlled manner, and consequently, the literature contains predominantly single-institutional retrospective series. All of these series span very long eras, and therefore the imaging, surgical, and radiotherapeutic techniques are extremely heterogeneous. Although reports in the literature have generally demonstrated improved outcomes with the use of immediate adjuvant RT, none of the retrospective series utilize predefined criteria for selecting adjuvant RT, thereby introducing enormous bias and making data interpretation even more complicated. ${ }^{2,3,5-7}$ An initial report from MDACC described the outcomes of 51 patients with spinal MPE and demonstrated that adjuvant RT significantly improved local control (LC) after STR (10-year LC was 0\% after STR alone vs 65\% with adjuvant RT, $p=0.008$ ), as well as following GTR (median LC was 4.75 years after GTR alone vs 10.5 years 
with adjuvant $\mathrm{RT}, \mathrm{p}=0.03$ ). These data were subsequently combined in a larger pooled analysis of 183 patients with spinal MPE by Weber and colleagues, ${ }^{2}$ in which $47 \%$ of patients received adjuvant RT. In their multivariable analysis, after accounting for age, extent of resection, and metastasis at diagnosis, treatment with surgery and adjuvant RT was associated with both improved LC (standarddose RT: HR 0.34, 95\% CI 0.15-0.77; high-dose RT: HR $0.34,95 \%$ CI $0.16-0.71 ; \mathrm{p}<0.001)$ and progression-free survival (standard-dose RT: HR 0.28, 95\% CI 0.12-0.60; high-dose RT: HR 0.28, 95\% CI 0.14-0.57; p < 0.001). Ten-year progression-free survival was $70 \%$ with adjuvant RT compared to $<40 \%$ with surgery alone, but no differences were specifically reported regarding extent of resection and effect of RT. ${ }^{2}$ In contrast, we found no significant benefit in RFS with adjuvant RT in both the initial and present analysis, regardless of extent of resection. ${ }^{9}$ Although both studies had similar follow-up periods and roughly the same proportion of patients achieving GTR (54\%-66\%), key differences in selection criteria for adjuvant RT in both centers may lead to significant selection bias. For example, of the 10 patients treated with adjuvant RT in this series, 5 had a GTR, whereas one-third of STR patients (the remaining 5 patients) received adjuvant RT. Therefore, our series could have limited power for demonstrating the benefit given that half of the patients who received adjuvant treatment received it after GTR and therefore were in the group least likely to benefit from RT. This hypothesis was bolstered by the finding that after enriching the group with patients treated with adjuvant RT at recurrence, the benefit of RT was more easily demonstrated. Therefore, high-risk groups (STR or questionable GTR) should still be evaluated and considered for postoperative RT given the risk of disease recurrence and potential benefits of adjuvant treatment. It is important to note that pediatric patients were not well represented in this series, and as other series demonstrate a higher risk of recurrence in the pediatric population, even in the setting of GTR, our results should not be extrapolated to pediatric patients. ${ }^{10}$

A unique finding from our series is that at first recurrence, treatment with RT improves median RFS by almost 8 years, from 1.6 years with surgery alone to 9.5 years with the addition of RT. Thus, our data suggest that for patients who do not receive RT in the adjuvant setting, salvage RT is crucial in reducing the risk of further recurrence, even after salvage GTR. Although we were unable to report toxicity data, Weber and colleagues demonstrated a trend toward increased late adverse events with surgery plus RT compared with surgery alone ( $43.9 \%$ vs $56.1 \%$, respectively; $\mathrm{p}=0.07){ }^{2}$ However, in light of recent advances in RT techniques that minimize the dose to surrounding critical structures and associated toxicity, such as proton therapy, the therapeutic ratio may shift to favor upfront adjuvant therapy as opposed to salvage treatment. ${ }^{11-15}$ Molecular characterization of these tumors is key to shedding some light as to whether there are distinct molecular entities which are likely to recur, as patients in this series experienced up to 4 episodes of disease relapse, despite similar initial pathologies.

Distant spinal failure as a component of disease recurrence is relatively common. In our series, $25 \%$ of failures were distant. No obvious trend emerged regarding the location of distant failures as they were found to be located either adjacent, nonadjacent, cranial, or caudal to the site of initial disease. In the study by Weber and colleagues, $29 \%$ had distant spine failure and $19 \%$ had brain failure. ${ }^{2}$ A recent series of 19 patients reported that $58 \%$ of patients had distant disease, $36 \%$ of which was present at diagnosis. ${ }^{16}$ Weber et al. further note that given the indolent clinical course of asymptomatic distant disease, close observation is a reasonable strategy. Given the high rates of distant failure, surveillance imaging of the entire neuraxis is a crucial component of disease management.

\section{Study Limitations}

It is important to note the limitations of the current analysis, including its retrospective nature and small sample size. Because the study spanned 4 decades, advances in imaging (CT to MRI), surgery, and RT (3D conformal to intensity-modulated RT) occurred. Management was subject to selection bias and follow-up schedules were inconsistent. Treatment outcomes outside of recurrencespecific data, such as recovery from surgery or presence or absence of neurological deficits at the time of initial surgery or at disease relapse, would be useful to include given the comparison across modalities (surgery with or without RT); however, these variables were not studied in this analysis. Additionally, we were unable to report toxicity data. Despite these limitations, our data represent an important contribution to the literature for this rare entity, given the long-term follow-up and meticulous assessment of initial surgery and recurrence patterns, which cannot easily be performed in larger database studies.

\section{Conclusions}

Owing to the relatively young age at presentation and long natural history of spinal MPE, our findings support aggressive initial surgical resection to provide the longest recurrence-free interval and highlight the benefit of RT at the time of salvage for those patients treated with surgery alone in the upfront setting. Surveillance imaging of the entire neuraxis is crucial as distant failure is not uncommon.

\section{References}

1. Bates JE, Choi G, Milano MT. Myxopapillary ependymoma: a SEER analysis of epidemiology and outcomes. J Neurooncol. 2016;129(2):251-258.

2. Weber DC, Wang Y, Miller R, et al. Long-term outcome of patients with spinal myxopapillary ependymoma: treatment results from the MD Anderson Cancer Center and institutions from the Rare Cancer Network. Neuro Oncol. 2015;17(4):588-595.

3. Klekamp J. Spinal ependymomas. Part 2: Ependymomas of the filum terminale. Neurosurg Focus. 2015;39(2):E7.

4. Sonneland PRL, Scheithauer BW, Onofrio BM. Myxopapillary ependymoma. A clinicopathologic and immunocytochemical study of 77 cases. Cancer. 1985;56(4):883-893.

5. Akyurek S, Chang EL, Yu TK, et al. Spinal myxopapillary ependymoma outcomes in patients treated with surgery and radiotherapy at M.D. Anderson Cancer Center. J Neurooncol. 2006;80(2):177-183. 
6. Pica A, Miller R, Villà S, et al. The results of surgery, with or without radiotherapy, for primary spinal myxopapillary ependymoma: a retrospective study from the rare cancer network. Int J Radiat Oncol Biol Phys. 2009;74(4):1114-1120.

7. Tsai CJ, Wang Y, Allen PK, et al. Outcomes after surgery and radiotherapy for spinal myxopapillary ependymoma: update of the MD Anderson Cancer Center experience. Neurosurgery. 2014;75(3):205-214.

8. Pesce A, Palmieri M, Armocida D, et al. Spinal myxopapillary ependymoma: the Sapienza University experience and comprehensive literature review concerning the clinical course of 1602 patients. World Neurosurg. 2019;129:245-253.

9. Chao ST, Kobayashi T, Benzel E, et al. The role of adjuvant radiation therapy in the treatment of spinal myxopapillary ependymomas. J Neurosurg Spine. 2011;14(1):59-64.

10. Feldman WB, Clark AJ, Safaee M, et al. Tumor control after surgery for spinal myxopapillary ependymomas: distinct outcomes in adults versus children: a systematic review. J Neurosurg Spine. 2013;19(4):471-476.

11. Baumann BC, Lustig RA, Mazzoni S, et al. A prospective clinical trial of proton therapy for chordoma and chondrosarcoma: Feasibility assessment. J Surg Oncol. 2019;120(2):200-205

12. Brown AP, Barney CL, Grosshans DR, et al. Proton beam craniospinal irradiation reduces acute toxicity for adults with medulloblastoma. Int J Radiat Oncol Biol Phys. 2013;86(2):277-284.

13. MacEwan I, Chou B, Moretz J, et al. Effects of vertebralbody-sparing proton craniospinal irradiation on the spine of young pediatric patients with medulloblastoma. Adv Radiat Oncol. 2017;2(2):220-227.

14. Paganetti H, Giantsoudi D. Relative biological effectiveness uncertainties and implications for beam arrangements and dose constraints in proton therapy. Semin Radiat Oncol. 2018;28(3):256-263.

15. Yoon M, Shin DH, Kim J, et al. Craniospinal irradiation techniques: a dosimetric comparison of proton beams with standard and advanced photon radiotherapy. Int J Radiat Oncol Biol Phys. 2011;81(3):637-646.
16. Kraetzig T, McLaughlin L, Bilsky MH, Laufer I. Metastases of spinal myxopapillary ependymoma: unique characteristics and clinical management. J Neurosurg Spine. 2018;28(2):201-208.

\section{Disclosures}

Dr. Kotecha reports receiving honoraria from Elekta AB, Accuray Inc., and Novocure Inc. Dr. Tom reports receiving research support from Blue Earth Diagnostics. Dr. Krishnaney reports being a consultant for Stryker. Dr. Steinmetz reports receiving royalties from Zimmer Biomet and honoraria from Stryker and Globus, and being a consultant for Intellirod. Dr. Suh reports being a consultant for Abbvie. Dr. Chao reports receiving honoraria from Varian Medical Systems.

\section{Author Contributions}

Conception and design: Kotecha. Acquisition of data: Kotecha, Reddy. Analysis and interpretation of data: Chao, Kotecha, Tom, Naik, Reddy. Drafting the article: Kotecha, Tom, Naik, Reddy. Critically revising the article: all authors. Reviewed submitted version of manuscript: Chao, Kotecha, Naik, Angelov, Benzel, Reddy, Prayson, Kalfas, Schlenk, Krishnaney, Steinmetz, Bingaman, Suh. Approved the final version of the manuscript on behalf of all authors: Chao. Statistical analysis: Reddy.

\section{Supplemental Information Online-Only Content}

Supplemental material is available with the online version of the article.

Supplemental Tables 1 and 2. https://thejns.org/doi/suppl/ 10.3171/2020.2.SPINE191534.

\section{Correspondence}

Samuel T. Chao: Taussig Cancer Institute, Cleveland Clinic, Cleveland,OH.chaos@ccf.org. 